\title{
Diagnostic Value of TB-IGRA, PPD, TB-DNA-PCR and ADA in Tuberculous Pleural Effusion
}

\author{
Weijia Lin ${ }^{*}$, Zhi Liu², Yaping Zhang1, Yanan Yu1, Yang Liu1 ${ }^{1}$, Ping Feng1, Feng Li' ${ }^{1}$ Zhihua Zhang1\# \\ ${ }^{1}$ Department of Respiratory Medicine, The First Affiliated Hospital of Hebei North University, Zhangjiakou, China \\ ${ }^{2}$ Department of Orthopaedics, The First Affiliated Hospital of Hebei North University, Zhangjiakou, China \\ Email: lwj1987lu@163.com, "ZZH19641229@163.com
}

How to cite this paper: Lin, W.J., Liu, Z., Zhang, Y.P., Yu, Y.N., Liu, Y., Feng, P., Li, F. and Zhang, Z.H. (2021) Diagnostic Value of TB-IGRA, PPD, TB-DNA-PCR and ADA in Tuberculous Pleural Effusion. Journal of Tuberculosis Research, 9, 124-130. https://doi.org/10.4236/jtr.2021.93012

Received: June 10, 2021

Accepted: July 23, 2021

Published: July 26, 2021

Copyright $\odot 2021$ by author(s) and Scientific Research Publishing Inc. This work is licensed under the Creative Commons Attribution International License (CC BY 4.0).

http://creativecommons.org/licenses/by/4.0/ (c) (i) Open Access

\begin{abstract}
Objective: To investigate the clinical diagnostic value of TB-IGRA (Tuberculosis-Interferon Gamma Release Assay), PPD (Intradermal Tuberculin Test), TB-DNA-PCR (Tuberculosis-Deoxyribonucleic-Polymerase Chain Reaction) and $\mathrm{ADA}$ (Adenosine Aeaminase) in tuberculous pleural effusion. Methods: 60 patients with tuberculous pleural effusion discharged from our department from January 1, 2018 to December 31, 2019 were selected. Moreover, the TBIGRA in peripheral blood, PPD test, TB-DNA-PCR and ADA in pleural effusion were detected. Subsequently, the positive rate, negative rate, sensitivity and omission diagnostic rate of TB-IGRA, PPD, TB-DNA-PCR, ADA and combined TB-IGRA were calculated. Results: The positive rate and sensitivity of TB-IGRA, PPD, TB-DNA-PCR, and ADA were $95 \%, 71.67 \%, 5 \%$ and $86.67 \%$ respectively. The omission diagnostic rate was $5 \%, 28.33 \%, 95 \%$ and 13.33\%. TB-IGRA showed the highest positive rate and sensitivity, and TBDNA-PCR represented the highest omission diagnostic rate. The sensitivity of TB-IGRA + PPD was $98.33 \%$, while the omission diagnostic rate was $51.67 \%$. The sensitivity of TB-IGRA + TB-DNA-PCR was $95 \%$, while the omission diagnostic rate was $5 \%$. The sensitivity of TB-IGRA + ADA was $100 \%$, while the omission diagnostic rate was $0 \%$. In addition, the TB-IGRA + ADA had the highest sensitivity and the lowest omission diagnostic rate. Conclusion: TBIGRA has high positive rate, high sensitivity and low omission diagnostic rate, which is superior to the traditional sputum test for tuberculosis. Notably, the combination of PPD, TB-DNA-PCR, ADA is capable of improving the diagnosis rate, and the diagnosis rate can reach $100 \%$ when combined with ADA, which is able to provide solid diagnostic value in clinical practice.
\end{abstract}

\section{Keywords}

TB-IGRA, PPD, TB-DNA-PCR, ADA, Diagnosis 


\section{Introduction}

Pleural effusion is one of the common clinical diseases. In 2007, a multicenter investigation in the United States has revealed that the number of patients with pleural effusion is 1.5 million every year. Congestive heart failure is the most common cause, followed by parapneumonic pleural effusion and malignant pleural effusion. In the prospective single center clinical study among the British population by Walker et al. [1], infection and heart failure are the most common causes of nonmalignant pleural effusion. Moreover, according to the Taghizadeh et al. [2] retrospective analysis of the patient information in the national inpatient sampling (NIS) database system of the United States, malignant pleural effusion has been considered as a common and expensive disease in the United States. However, the overall data analysis of pleural effusion etiology, diagnosis and treatment, disease load in other countries is relatively less. In 2017, Beijing Chaoyang Hospital [3] conducted a retrospective single center clinical study on the Etiological Distribution of pleural effusion, involving a total of 1541 patients. The results revealed that the bacterial infection, malignant tumor, cardiac insufficiency and tuberculosis were the most common causes. In addition, the Mi yun teaching hospital of Capital Medical University [4] analyzed the etiology of 67 patients admitted with unilateral pleural effusion from January 2016 to December 2017, including 24 cases of tuberculous pleurisy, 11 cases of cardiac insufficiency, 18 cases of malignant pleural effusion, 3 cases of renal insufficiency and 11 cases of pneumonia. Tuberculosis and pneumonia are the main causes in developing countries [5], it found that the most common causes in developed countries are cardiac insufficiency, pneumonia and malignant tumor. As we all know, Zhangjiakou is a poor mountain area with a high incidence rate of tuberculous pleurisy, the phenomenon of returning to poverty due to illness is very common, therefore, early diagnosis plays a conducive role in early treatment with improving the cure rate, reducing the patients and social economic burden. Large data samples suggested that TB-IGRA had high sensitivity, accuracy and specificity in tuberculous pleural. However, since the traditional PPD, TB-DNA-PCR, ADA detection methods are low in sensitivity, it is urgent to find faster methods with higher sensitive, specific for diagnostic of tuberculous pleural.

\section{Materials and Methods}

\subsection{Materials}

From January 1, 2018 to December 31, 2019, 60 discharged cases with tuberculous pleural effusion were selected.

\subsection{Inclusion and Exclusion Criteria}

Inclusion criteria: 1) discharge time: January 1, 2018 to December 31, 2019; 2) Length of hospitalization: $>24$ hours; 3 ) The patient age: $\geq 18$ years old; 4) Discharge diagnosis: Tuberculous pleurisy.

Exclusion criteria: 1) No pleural effusion confirmed by imaging; 2) Patients 
discharged within 24 hours; 3 ) For those who were hospitalized repeatedly due to pleural effusion, only the data of the first hospitalization in this year were collected; 4) Discharge diagnosis was non tuberculous pleurisy.

\section{Methods}

All patients were tested by four detection methods of tuberculosis: TB-IGRA, PPD, TB-DNA-PCR and ADA.

\subsection{TB-IGRA}

In the morning, 6 to $10 \mathrm{~mL}$ of fasting peripheral venous blood was extracted under anticoagulation with heparin, meanwhile, the peripheral monocytes were isolated. In the four wells (blank control, test well A, test well B and positive control) of the microplate, $50 \mu \mathrm{L}$ cell culture medium, ESAT- 6 (antigen A), CFP10 (antigen B), positive quality control solution, and then each well was added with $100 \mu \mathrm{L}$ samples, all the wells were incubated in incubator. The microplate was washed with PBS, and then the enzyme labeled antibody was added into the microplate. Then the microplate was washed, and substrate reaction solution was added into the microplate. ELISPOT reader was used to read and count the number of spots, and the result was evaluated by following the instructions of the kit strictly. If the number of negative spots is ranged from 0 to 5 , and the spots number of antigen A or antigen B test wells minus the spots number of negative wells is greater than 6 , it will be considered as positive results. While, if the number of negative spots is more than 6 , the number of test spots must be more than 2 times of the number of negative spots, it will be considered as positive results. If the above standards are not met and the positive control well is normal, the test result will be negative.

\subsection{PPD}

After subcutaneous injection of $5 \mathrm{IU}$ tuberculosis protein derivative into the medial side of left forearm for 72 hours, the average diameter of induration was calculated by (transverse diameter + longitudinal diameter)/2. If there is no induration or the average diameter of induration is less than $10 \mathrm{~mm}$, it is judged as negative (-); if it is more than $10 \mathrm{~mm}$, it is judged as positive $=(+)$.

\subsection{Pleural Effusion TB-DNA-PCR}

Drainage of pleural effusion was placed in sterile sputum box for examination. TB-DNA detection kit of Sun Yat-sen University Da'an gene Co., Ltd. was adopted, and the detection process was operated according to the instructions.

\subsection{Pleural Effusion ADA}

$5 \mathrm{ml}$ of pleural effusion drainage stored in the sterile sputum box was detected. The test kit of Beijing Century ward Biotechnology Co., Ltd. was adopted, and the detection process was operated according to the instructions. 


\subsection{Observation Index [6]}

The positive rate, negative rate, sensitivity and omission diagnostic rate of TBIGRA, PPD, TB-DNA-PCR, ADA and combined with TB-IGRA.

\subsection{Statistical Method [7] [8]}

SPSS 20.0 statistical software was employed to process data. The measurement data were showed by $\bar{x} \pm s$, while the enumeration data were represented in\%.

\section{Results}

\section{1) General results}

In 60 cases of tuberculous pleural effusion, there were 27 males and 33 females, aged from 19 to 86 years old, with an average age of (59.48 $1 \pm 13.732)$.

2) The positive and negative rates of TB-IGRA, PPD, TB-DNA-PCR and ADA were compared

A total of 60 cases were included in this study. The positive cases of TB-IGRA were 57 cases (95\%), and the negative cases were 3 cases (5\%). The positive cases of PPD were 43 cases (71.67\%), and the negative rate was 17 cases $(28.33 \%)$. The TB-DNA-PCR positive cases were 3 cases (5\%), and negative cases were 57 cases (95\%). The positive cases of ADA were 52 cases (86.67\%), and the negative cases were 8 cases $(13.33 \%)$ (Table 1, Figure 1 ).

3) The sensitivity and omission diagnostic rate of TB-IGRA, PPD, TB-DNA-PCR, ADA and combined with TB-IGRA were compared

The sensitivity of TB-IGRA was $95 \%$ while the omission diagnostic rate was $5 \%$. The sensitivity of PPD was $71.67 \%$ while the omission diagnostic rate was $28.33 \%$. The sensitivity of TB-DNA-PCR was $5 \%$ while the omission diagnostic rate was $95 \%$. The sensitivity of ADA was $86.67 \%$ while the omission diagnostic rate was $13.33 \%$. TB-IGRA showed the highest sensitivity and TB-DNA-PCR represented

Table 1. The positive rate and negative rate of four tuberculosis detection indexes.

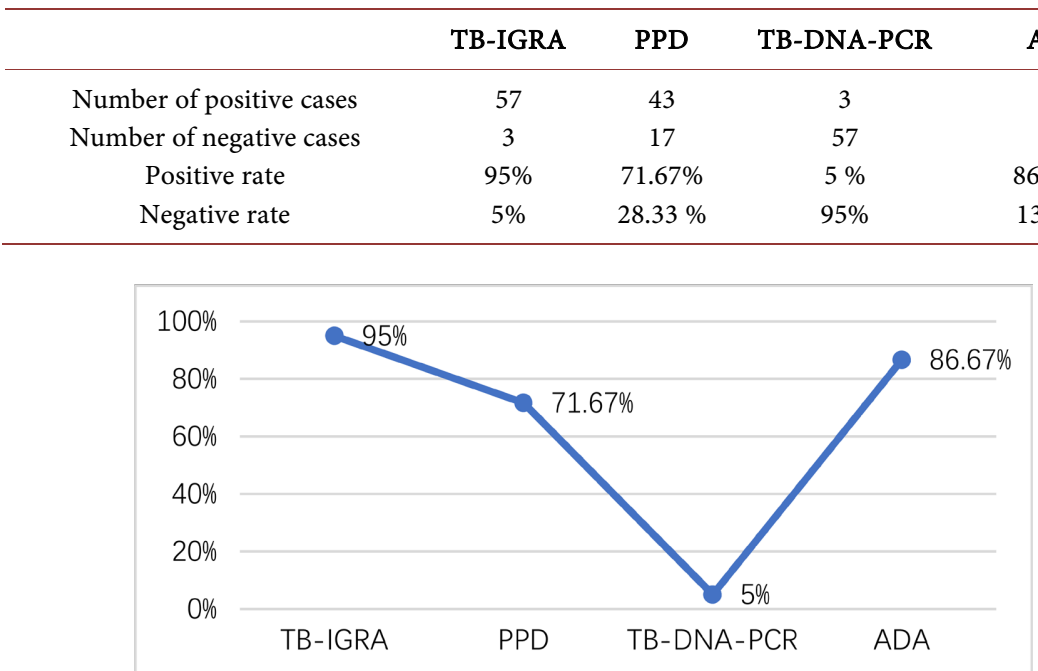

Figure 1. The positive rate and negative rate of four tuberculosis detection indexes 
the highest omission diagnostic rate.

The sensitivity of TB-IGRA + PPD combination detection was $98.33 \%$ while the omission diagnostic rate was $51.67 \%$. The sensitivity of TB-IGRA + TB-DNAPCR was $95 \%$ while the omission diagnostic rate was $5 \%$. The sensitivity of TBIGRA + ADA was $100 \%$ while the omission diagnostic rate was $0 \%$. Notably, the TB-IGRA + ADA showed the highest sensitivity and the lowest omission diagnostic rate. (Table 2, Figure 2 and Figure 3)

Table 2. Sensitivity and omission diagnostic rate of four tuberculosis detection indexes (\%)

\begin{tabular}{ccc}
\hline & sensitivity & omission diagnostic rate \\
\hline TB-IGRA & $95(57 / 60)$ & $5(3 / 60)$ \\
PPD & $71.67(43 / 60)$ & $28.33(17 / 60)$ \\
TB-DNA-PCR & $5(3 / 60)$ & $95(57 / 60)$ \\
ADA & $86.67(52 / 60)$ & $13.33(8 / 60)$ \\
TB-IGRA+PPD & $98.33(59 / 60)$ & $1.67(1 / 60)$ \\
TB-IGRA + TB-DNA-PCR & $95(57 / 60)$ & $5(3 / 60)$ \\
TB-IGRA+ADA & $100(60 / 60)$ & $0(0 / 60)$ \\
\hline
\end{tabular}

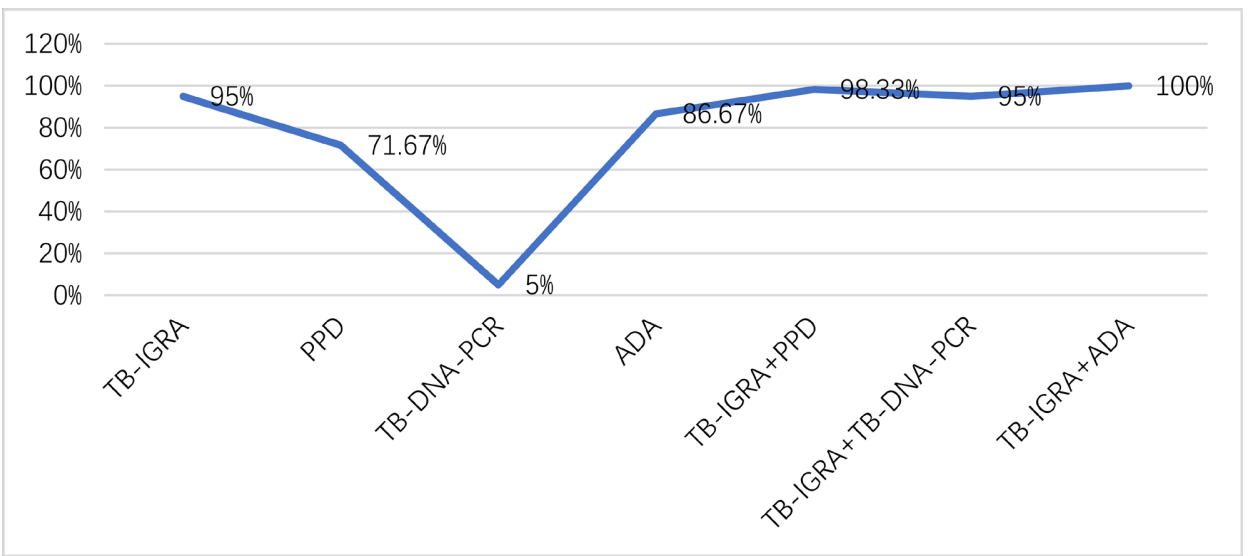

Figure 2. Sensitivity of four tuberculosis detection indexes.

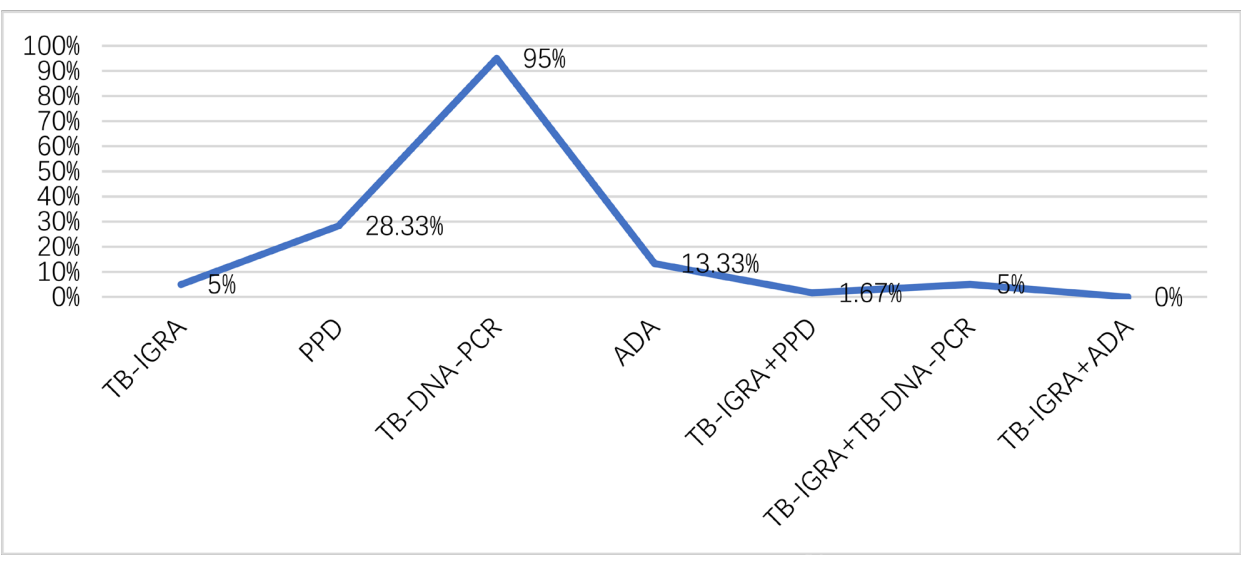

Figure 3. The omission diagnostic rate of four tuberculosis detection indexes. 


\section{Conclusions}

Tuberculous pleural effusion is a type of pulmonary tuberculosis [9] [10], which is caused by Mycobacterium tuberculosis invading the pleura [11], the main clinical manifestations of the patients are chest tightness, shortness of breath, chest pain, low-grade fever, perspire during sleep, and the gold standard for the diagnosis of which is detecting the tuberculosis in pleural effusion, however, the positive rate of this method is low, most of the diagnosis depends on clinical manifestations. [12]. Traditional diagnostic methods for pulmonary tuberculosis have the characteristics of low sensitivity and unsatisfying specificity. For example, the culture time of Mycobacterium tuberculosis is long and the positive rate is low, and tuberculin test is prone to be a false positive or false negative results. The detection of Mycobacterium tuberculosis specific cellular immune response (TB-IGRA) [13] is a diagnostic method of tuberculosis developed in recent years, which has been proved to be a sensitive and specific detection method in Mycobacterium tuberculosis infection, especially, it can be early diagnosed before bacteriological results and typical imaging findings. Therefore, it has been employed as a common method for tuberculosis detection in European and American countries. Moreover, it has been recorded into the 2018 edition of the guideline for TB diagnosis [14] [15]. In addition, many studies have shown that [16] TB-IGRA has represented a satisfying diagnostic efficacy in pulmonary tuberculosis. Notably, combined with classical PPD, TB-DNA-PCR and ADA, it is capable of improving the positive rate of diagnosis and reducing the rate of the omission diagnostic rate.

A total of 60 cases were included in this study. The positive rate and sensitivity of TB-IGRA, PPD, TB-DNA-PCR and ADA were $95 \%, 71.67 \%, 5 \%$ and $86.67 \%$ respectively. The rate of the omission diagnostic rate was $5 \%, 28.33 \%, 95 \%$ and 13.33\%. TB-IGRA showed the highest positive rate and sensitivity, and TB-DNAPCR represented the highest omission diagnostic rate. The sensitivity of TB-IGRA + PPD was $98.33 \%$ while the omission diagnostic rate was $51.67 \%$. The sensitivity of TB-IGRA + TB-DNA-PCR was $95 \%$ while the omission diagnostic rate was $5 \%$. The sensitivity of IGRA + ADA was $100 \%$ while the omission diagnostic rate was $0 \%$. TB-IGRA + ADA showed the highest sensitivity and the lowest omission diagnostic rate.

In conclusion, TB-IGRA shows the high positive rate, high sensitivity and low omission diagnostic rate relatively, which is more satisfying and applicable than the traditional sputum examination of tuberculosis. When it is combined with PPD, TB-DNA-PCR、ADA, the diagnosis rate can be improved, when combined with ADA, the diagnosis accuracy can reach $100 \%$, which has made up for their own shortcomings and represented a promising clinical application value in the auxiliary diagnosis of tuberculous pleurisy.

\section{Project Name}

2018 Key project plan of medical science research in Hebei Province 20180869, 
2021Key R \& D plan of Zhangjiakou City, Hebei Province 2021046D.

\section{Conflicts of Interest}

The authors declare no conflicts of interest regarding the publication of this paper.

\section{References}

[1] Walker, S.P., Morley, A.J., Stadon, L., et al. (2017) Nonmalignant Pleural Effusions: A Prospective Study of 356 Consecutive Unselected Patients. Chest, 151, 1099-1105. https://doi.org/10.1016/j.chest.2016.12.014

[2] Taghizadeh, N., Fortin, M. and Tremblay, A. (2017) US Hospitalizations for Malignant Pleural Effusions: Data from the 2012 National Inpatient Sample. Chest, 151, 845-854. https://doi.org/10.1016/j.chest.2016.11.010

[3] Wu, Y.-B., Du, Y., Lu, Y., et al. (2017) Etiology of Pleural Effusions. Chinese Journal of Respiratory and Critical Care Medicine, 16, 490-494.

[4] Zhang, E.H. (2019) Analysis of Etiology of 67 Patients with Pleural Effusion. China Medicine and Pharmacy, 9, 252-254.

[5] Corcoran, J.P., Hallifax, R. and Rahman, N.M. (2013) Advances in the Management of Pleural Disease. Expert Review of Respiratory Medicine, 7, 499-513. https://doi.org/10.1586/17476348.2013.838016

[6] Deng, A.-F., Zhang, G.-H., Lai, M., et al. (2021) Diagnostic Value of T Cell Spot Test of Tuberculosis Infection of Pleural Effusion and Peripheral Blood in Tuberculous Pleural Effusion. China Modern Medicine, 28, 145-148.

[7] Li, L.J. (2021) Clinical Significance of Combined Detection of T-SPOT.TB, PPD and $\mathrm{TB}-\mathrm{AB}$ in the Diagnosis of Active Pulmonary Tuberculosis. Occupational Health and Damage, 36, 106-109.

[8] Huang, Y.-N. (2021) Diagnostic Value of Parallel Detection of T-SPOT.TB, TB Sputum Smear and TB-DNA in Tuberculosis. Heilongjiang Medicine Journal, 34, 455-457.

[9] National Health Commission of the People's Republic of China (2017) WS 288-2017 Diagnosis for Pulmonary Tuberculosis Standards.

[10] National Health Commission of the People's Republic of China (2017) WS 196-2017 Classification of Tuberculosis.

[11] Chen, X.-Y. (2017) Diagnosis and Prospect of Tuberculous Pleurisy. Chinese Journal of Antituberculosis, 14, 1153-1156.

[12] Yin, X.-B., Tian, Q., Tian, S.-F., et al. (2017) The Value of T Cell Spot Test in the Diagnosis of Tuberculous Pleurisy. International Journal of Respiration, 37, 961-965.

[13] Sun, G.-Y., Zhao, G. and Gao, S.-L. (2020) Application of T-SPOT.TB Combined with Gene Chip Detection in the Diagnosis of Senile Pulmonary Tuberculosis. Chinese Journal of Gerontology, 40, 1851-1853.

[14] Suarez, I., Funger, S.M., Kroger, S., et al. (2019) The Diagnosis and Treatment of Tuberculosis. Deutsches Aerzteblatt International, 116, 729-735. https://doi.org/10.3238/arztebl.2019.0729

[15] Alnourtms. Smear Microscopy as a Diagnostic Tool Of tuberculosis; Review of Smear Negative Cases, Frequency, Risk Factors, and Prevention Criteria. India J Tuberc, 2018, 65, 190-194. https://doi.org/10.1016/j.ijtb.2018.02.001

[16] Luo, H.-H., Liu, Y.-L. and Luo, Y.-Q. (2021) The Diagnostic Value of T-SPOT. TB Combined with Pleural Effusion Adenosine Deaminase in Tuberculous Pleurisy. Guangzhou Medical Journal, 52, 109-102. 